\title{
Are There Magnetars in High Mass X-ray Binaries? The Case of SuperGiant Fast X-Ray Transients
}

\author{
E. Bozzo ${ }^{* a, b}$ M. Falanga ${ }^{c}$ L. Stella ${ }^{a}$ \\ a INAF - Osservatorio Astronomico di Roma, Via Frascati 33, 00044 Rome, Italy \\ ${ }^{b}$ Dipartimento di Fisica - Università di Roma “Tor Vergata”, via della Ricerca Scientifica 1, \\ 00133 Rome, Italy \\ ${ }^{c}$ CEA Saclay, DSM/DAPNIA/Service d'Astrophysique (CNRS FRE 2591), F-91191, Gif sur \\ Yvette, France
}

\begin{abstract}
In this paper we summarize some aspects of the wind accretion theory in high mass X-ray binaries hosting a magnetic neutron star and a supergiant companion. In particular, we concentrate on the different types of interaction between the inflowing wind matter and the neutron star magnetosphere that are relevant when accretion of matter onto the neutron star surface is largely inhibited; these include inhibition by the centrifugal and magnetic barriers. We show that very large luminosity swings ( $\sim 10^{4}$ or more on time scales as short as hours) can result from transitions across different regimes. This scenario is then applied to the activity displayed by supergiant fast X-ray transients (SFXTs), a new class of high mass X-ray binaries in our galaxy recently discovered with INTEGRAL. According to this interpretation we argue that SFXTs which display very large luminosity swings and host a slowly spinning neutron star are expected to be characterized by magnetar-like fields. Supergiant fast X-ray transients might thus provide a unique opportunity to detect and study accreting magnetars in binary systems.
\end{abstract}

7th INTEGRAL Workshop

September 8-11 2008

Copenhagen, Denmark

\footnotetext{
*Speaker.
} 


\section{Contents}

$\begin{array}{lr}\text { 1. Introduction } & 2\end{array}$

2. Stellar wind accretion $\quad 3$

$\begin{array}{lll}\text { 3. Transitions and paths across different regimes } & 7\end{array}$

4. Application to SFXT sources 9

$\begin{array}{lll}\text { 5. Conclusions } & 10\end{array}$

\section{Introduction}

Supergiant Fast X-ray transients (SFXTs) are a new class of high mass X-ray binaries (HMXB), recently discovered with INTEGRAL. These sources are observed to exhibit sporadic outbursts, lasting from minutes to hours, with peak X-ray luminosities between $\sim 10^{36}$ and $10^{37} \mathrm{erg} \mathrm{s}^{-1}$ (Sguera et al., 2005). No firm orbital period measurement has been obtained yet ${ }^{1}$. A recent list of confirmed $(\sim 5)$ and candidate $(\sim 6)$ SFXTs is given by Walter \& Zurita Heras (2007). Between outbursts, SFXTs remain in quiescence with luminosities in the range $\sim 10^{31}-10^{33} \mathrm{erg} \mathrm{s}^{-1}$. In some cases, very high peak-to-quiescence X-ray luminosity swings (factor of $\sim 10^{4}-10^{5}$ ) have been seen on timescales comparable to the outburst duration (e.g., In't Zand, 2005). Some SFXTs showed also flare-like activity at intermediate luminosity levels (e.g., Walter et al., 2006). Optical identifications of SFXTs show that these sources are associated to OB supergiant companion stars (see e.g., Walter \& Zurita Heras, 2007, and reference therein). These stars have typically a mass of $\mathrm{M}_{*} \sim 30 \mathrm{M}_{\odot}$, optical luminosity of $\log \left(\mathrm{L}_{*} / \mathrm{L}_{\odot}\right) \sim 5-6$, mass loss rate of $\dot{M}_{\mathrm{w}}=10^{-7}-10^{-5} \mathrm{M}_{\odot} \mathrm{yr}^{-1}$, wind velocity of $\mathrm{v}_{\mathrm{w}} \sim 1000-2000 \mathrm{~km} \mathrm{~s}^{-1}$, and are persistent soft X-ray sources with luminosity around $\sim 10^{32} \mathrm{erg} \mathrm{s}^{-1}$ (Cassinelli et al., 1981).

It is widely believed that SFXTs contain neutron stars (NS) sporadically accreting matter from a supergiant companion, and in the prototypical SFXTs XTE J1739-302 (Sguera et al., 2006) and IGR J16479-4514 (Walter et al., 2006) some evidence has been reported for spin periods in the $\sim 1000-2000$ s range. Proposed models for SFXTs generally involve accretion onto a NS immersed in the clumpy wind of its supergiant companion (In't Zand, 2005; Walter \& Zurita Heras, 2007). However, if accretion onto the collapsed object of SFXTs takes place both in quiescence and outburst, then the corresponding X-ray luminosity swing, typically a factor of $\sim 10^{4}-10^{5}$, would require wind inhomogeneities with a very large density and/or velocity contrast (according to the standard

\footnotetext{
${ }^{1}$ Only IGR J11215-5952 showed recurrent flaring activity, with a periodicity of $\sim 165 \mathrm{~d}$. This is interpreted as outbursts from a systems with an unusually long orbital period (Sidoli et al., 2007). Thus Walter \& Zurita Heras (2007) excluded these sources from their SFXT list.
} 


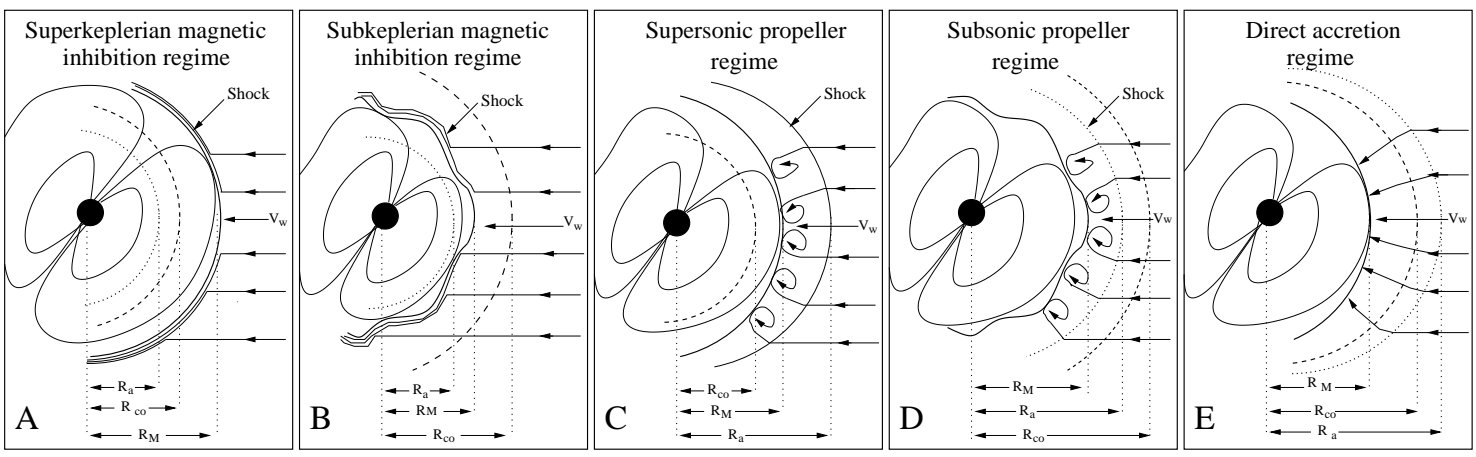

Figure 1: Schematic view of a magnetized NS interacting with the inflowing matter from its supergiant companion. All the regimes described in the text are shown, together with the relative position of the magnetospheric radius (solid line), the corotation radius (dashed line), and the accretion radius (dotted line). A wavy solid line is used when the magnetospheric boundary at the magnetospheric radius is Kelvin-Helmholtz unstable. In the supersonic and subsonic propeller regime convective motions at the base of the atmosphere are represented with small eddies.

wind accretion, the mass capture rate onto the NS scales like $\dot{M}_{\mathrm{w}} \mathrm{v}_{\mathrm{w}}^{-4}$ Davidson \& Ostriker, 1979). Models involving accretion of extremely dense clumps are still being actively pursued (Negueruela et al., 2008). The requirement on the density and/or velocity contrasts in the wind can be eased if there is a barrier that remains closed during quiescence, halting most of the accretion flow, and opens up in outbursts, leading to direct accretion. After reviewing briefly the theory of wind accretion in HMXBs ( $\$ 2$ and 3), we apply barrier scenarios to SFXTs in $\S 4$ and conclude that if SFXTs host slowly rotating NSs (spin periods of several hundreds to thousands seconds), then they must possess magnetar-like fields $\left(\sim 10^{14}-10^{15} \mathrm{G}\right)$.

\section{Stellar wind accretion}

We investigate here the conditions under which a magnetized NS can accrete matter from the wind of a massive companion. In the theory of wind accretion in HMXBs, the following radii are defined (e.g., Illarionov \& Sunyaev, 1975; Stella et al., 1986):

- The accretion radius, $R_{a}$ is the distance at which the inflowing matter is gravitationally focused toward the NS. It is usually expressed as

$$
R_{\mathrm{a}}=2 G M_{\mathrm{NS}} / v_{\mathrm{w}}^{2}=3.7 \times 10^{10} v_{8}^{-2} \mathrm{~cm},
$$

where $\mathrm{v}_{8}$ is the wind velocity in units of $1000 \mathrm{~km} \mathrm{~s}^{-1}$ and it is assumed that the orbital velocity of the star is negligible (Frank et al., 2002). Throughout the paper we fix the NS radius and mass at $\mathrm{R}_{\mathrm{NS}}=10^{6} \mathrm{~cm}$ and $\mathrm{M}_{\mathrm{NS}}=1.4 \mathrm{M}_{\odot}$, respectively. The fraction $\dot{M}_{\text {capt }} / \dot{M}_{\mathrm{w}}$ of the stellar wind mass loss rate $\left(\dot{M}_{\mathrm{w}}\right)$ captured by the NS depends on $\mathrm{R}_{\mathrm{a}}$ through (Frank et al., 2002)

$$
\dot{M}_{\text {capt }} / \dot{M}_{\mathrm{w}} \simeq R_{\mathrm{a}}^{2} /\left(4 a^{2}\right)=2 \times 10^{-5} v_{8}^{-4} a_{10 \mathrm{~d}}^{-2} .
$$

Here $\mathrm{a}=4.2 \times 10^{12} \mathrm{a}_{10 \mathrm{~d}} \mathrm{~cm}$ is the orbital separation, $\mathrm{a}_{10 \mathrm{~d}}=\mathrm{P}_{10 \mathrm{~d}}^{2 / 3} \mathrm{M}_{30}^{1 / 3}, \mathrm{P}_{10 \mathrm{~d}}$ is the binary orbital period in units of 10 days, and $\mathrm{M}_{30}$ is the total mass of the binary in units of $30 \mathrm{M}_{\odot}$ (we assumed circular orbits).

- The magnetospheric radius, $\mathrm{R}_{\mathrm{M}}$, at which the pressure of the NS magnetic field $\left(\mu^{2} /\left(8 \pi R_{\mathrm{NS}}^{6}\right)\right.$, 
with $\mu$ the NS magnetic moment) balances the ram pressure of the inflowing matter $\left(\rho_{\mathrm{w}} v_{w}^{2}\right)$. In the case in which $R_{M}>R_{a}$, the magnetospheric radius is given by (Davies \& Pringle, 1981)

$$
R_{\mathrm{M}}=3.3 \times 10^{10} \dot{M}_{-6}^{-1 / 6} v_{8}^{-1 / 6} a_{10 \mathrm{~d}}^{1 / 3} \mu_{33}^{1 / 3} \mathrm{~cm} .
$$

Here we assumed a non-magnetized spherically symmetric wind (Elsner \& Lamb, 1977), with density $^{2} \rho_{\mathrm{w}}\left(\mathrm{R}_{\mathrm{M}}\right) \sim \dot{M}_{\mathrm{w}} /\left(4 \pi \mathrm{a}^{2} \mathrm{v}_{\mathrm{w}}\right)$, a NS dipolar field with $\mu_{33}=\mu / 10^{33} \mathrm{G} \mathrm{cm}^{3}$, and $\dot{M}_{-6}=\dot{M}_{\mathrm{w}} / 10^{-6}$ $\mathrm{M}_{\odot} \mathrm{yr}^{-1}$. In the following sections we discuss the range of applicability of Eq. 2.3, and the regimes in which a different prescription for $\mathrm{R}_{\mathrm{M}}$ should be used.

- The corotation radius, $\mathrm{R}_{\mathrm{co}}$, at which the NS angular velocity equals the Keplerian angular velocity, i.e.

$$
R_{\mathrm{co}}=1.7 \times 10^{10} P_{\mathrm{s} 3}^{2 / 3} \mathrm{~cm} .
$$

Here $P_{\mathrm{s} 3}$ is the NS spin period in units of $10^{3} \mathrm{~s}$.

Changes in the relative position of these radii result into transitions across different regimes for the NS (Illarionov \& Sunyaev, 1975; Stella et al., 1986). In particular, the accretion radius and magnetospheric radius depend on the wind parameters (see Eqs. 2.1 and 2.3), which can vary on a wide range of timescales (from hours to months). Therefore, variations in the wind parameters can cause the NS to undergo transitions across different regimes on comparably short timescales, thus opening the possibility to explain the properties of some classes of highly variable X-ray sources through them. Below we summarise the different regimes of a magnetic rotating NS, subject to a varying stellar wind.

- Outside the accretion radius: the magnetic inhibition of accretion $\left(\mathbf{R}_{M}>\mathbf{R}_{\mathrm{a}}\right)$. In systems with $R_{M}>R_{a}$ the mass flow from the companion star interacts directly with the NS magnetosphere without significant gravitational focusing, forming a bow shock at $R_{M}$ (Harding et al., 1992; Toropina et al., 2006). At least in the front part of the shock, i.e. in the region around the stagnation point, the whole kinetic energy of the inflowing matter is converted into thermal energy. The power released in this region is of order

$$
L_{\text {shock }} \simeq \frac{\pi}{2} R_{\mathrm{M}}^{2} \rho_{\mathrm{w}} v_{\mathrm{w}}^{3}=4.7 \times 10^{29} R_{\mathrm{M} 10}^{2} v_{8}^{2} a_{10 \mathrm{~d}}^{-2} \dot{M}_{-6} \mathrm{erg} \mathrm{s}^{-1}
$$

$\left(\mathrm{R}_{\mathrm{M} 10}=\mathrm{R}_{\mathrm{M}} / 10^{10} \mathrm{~cm}\right)$, and is mainly radiated in the X-ray band. We distinguish two different regimes of magnetic inhibition of accretion:

- The superKeplerian magnetic inhibition regime: $\mathrm{R}_{\mathrm{M}}>\mathrm{R}_{\mathrm{a}}, \mathrm{R}_{\mathrm{co}}$. In this case the magnetospheric radius is larger than both the accretion and corotation radii. Matter that is shocked and halted close to $R_{M}$ cannot proceed further inward, due to the rotational drag of the NS magnetosphere which is locally superKeplerian. Since magnetospheric rotation is also supersonic, the interaction between the NS magnetic field and matter at $R_{M}$ results in rotational energy dissipation and thus, NS spin down. This process releases energy at a rate

$$
L_{\mathrm{sd}} \simeq \pi R_{\mathrm{M}}^{2} \rho_{\mathrm{w}} v_{\mathrm{w}}\left(R_{\mathrm{M}} \Omega\right)^{2} \simeq 3.7 \times 10^{29} R_{\mathrm{M} 10}^{4} \dot{M}_{-6} a_{10 \mathrm{~d}}^{-2} P_{\mathrm{s} 3}^{-2} \mathrm{erg} \mathrm{s}^{-1},
$$

which adds to the shock luminosity (Eq. 2.5).

\footnotetext{
${ }^{2}$ We approximated $a-R_{M} \simeq a$, which is satisfied for a very wide range of parameters.
} 
- The subKeplerian magnetic inhibition regime: $\mathrm{R}_{\mathrm{a}}<\mathrm{R}_{\mathrm{M}}<\mathrm{R}_{\mathrm{co}}$. In this case the magnetospheric drag is subKeplerian and matter can penetrate the NS magnetosphere through KelvinHelmholtz instabilities (KHI, Harding et al., 1992) and Bohm diffusion (Ikhsanov \& Pustil'nik, 1996). The mass inflow rate across $R_{M}$ resulting from the KHI depends on the efficiency factor $\eta_{\mathrm{KH}} \sim 0.1$, the shear velocity at $\mathrm{R}_{\mathrm{M}} \mathrm{v}_{\mathrm{sh}}$, and the densities $\rho_{\mathrm{i}}$ and $\rho_{\mathrm{e}}$ inside and outside the magnetospheric boundary at $R_{M}$, respectively. The luminosity released by accretion of this matter onto the NS is given by

$$
L_{\mathrm{KH}}=3.5 \times 10^{34} \eta_{\mathrm{KH}} R_{\mathrm{M} 10}^{2} a_{10 \mathrm{~d}}^{-2} \dot{M}_{-6}\left(\rho_{\mathrm{i}} / \rho_{\mathrm{e}}\right)^{1 / 2}\left(1+\rho_{\mathrm{i}} / \rho_{\mathrm{e}}\right)^{-1} \mathrm{erg} \mathrm{s}^{-1},
$$

or

$$
L_{\mathrm{KH}}=8.8 \times 10^{34} \eta_{\mathrm{KH}} P_{\mathrm{s} 3}^{-1} R_{\mathrm{M} 10}^{3} a_{10 \mathrm{~d}}^{-2} v_{8}^{-1} \dot{M}_{-6}\left(\rho_{\mathrm{i}} / \rho_{\mathrm{e}}\right)^{1 / 2}\left(1+\rho_{\mathrm{i}} / \rho_{\mathrm{e}}\right)^{-1} \mathrm{erg} \mathrm{s}^{-1},
$$

if the shear velocity is taken to be the gas velocity in the post-shock region close to $R_{M}$ or the rotational velocity of the NS magnetosphere, respectively (for simplicity, we consider throughout this paper $L_{K H}$ equal to the largest of the above two values). The ratio $\rho_{\mathrm{i}} / \rho_{\mathrm{e}}$ can be calculated from the equation of the mass conservation across the KHI unstable layer, i.e.

$$
R_{\mathrm{M}}^{2} \rho_{\mathrm{e}} v_{\text {conv }} \simeq R_{\mathrm{M}} h_{\mathrm{t}} \rho_{\mathrm{i}} v_{\mathrm{ff}}\left(R_{\mathrm{M}}\right),
$$

where $\mathrm{h}_{\mathrm{t}}$ is the height of the unstable layer (Burnard et al., 1983). Throughout this paper we assume $\mathrm{h}_{\mathrm{t}}=R_{\mathrm{M}}$ (Bozzo et al., 2008). The contribution of Bohm diffusion to the total mass inflow rate through the magnetosphere in the subKeplerian magnetic inhibition regime can be calculated according to Ikhsanov \& Pustil'nik (1996), and we found that it is orders of magnitude smaller than that due to the KHI over the whole range of parameters relevant to this work. Similarly, the contribution to the total luminosity resulting from the shock at the magnetospheric boundary can be neglected in this regime.

- Inside the accretion radius: $\mathbf{R}_{M}<\mathbf{R}_{\mathrm{a}}$. Once $\mathrm{R}_{\mathrm{M}}$ is inside the accretion radius, matter flowing from the companion star is shocked adiabatically at $\mathrm{R}_{\mathrm{a}}$ (this produces an almost negligible contribution to the total luminosity) and halted at the NS magnetosphere. In the region between $\mathrm{R}_{\mathrm{a}}$ and $\mathrm{R}_{\mathrm{M}}$ this matter redistributes itself into an approximately spherical configuration (resembling an "atmosphere"), whose shape and properties are determined by the interaction between matter and NS magnetic field at $R_{M}$ (Davies et al., 1979; Davies \& Pringle, 1981). A hydrostatic equilibrium ensues when radiative losses inside $R_{a}$ are negligible ${ }^{3}$; the atmosphere is stationary on dynamical timescales, and a polytropic law of the form $p \propto \rho^{1+1 / n}$ can be assumed for the pressure and density of the atmosphere. The value of the polytropic index $n$ depends on the conditions at the inner boundary of the atmosphere, and in particular on the rate at which energy is deposited there. Three different regimes can be distinguished:

- The supersonic propeller regime: $\mathrm{R}_{\mathrm{co}}<\mathrm{R}_{\mathrm{M}}<\mathrm{R}_{\mathrm{a}}$. In this case the rotational velocity of the NS magnetosphere at $R_{M}$ is supersonic; the interaction with matter in the atmosphere leads to dissipation of some of the star's rotational energy and thus spin-down. Turbulent motions are

\footnotetext{
${ }^{3}$ We checked this is verified for all the case of interest for this paper.
} 
generated at $R_{M}$ which convect this energy up through the atmosphere, until it is lost at its outer boundary. In this case $n=1 / 2$, and the balance between the magnetic and gas pressure gives

$$
R_{\mathrm{M}} \simeq 2.3 \times 10^{10} a_{10 \mathrm{~d}}^{4 / 9} \dot{M}_{-6}^{-2 / 9} v_{8}^{4 / 9} \mu_{33}^{4 / 9} \mathrm{~cm} .
$$

Matter that is shocked at $\sim R_{a}$, reaches the magnetospheric boundary at $R_{M}$ where the interaction with the NS magnetic field draws energy from NS rotation. According to Davies \& Pringle (1981), this gives the largest contribution to the total luminosity in this regime

$$
\begin{aligned}
L_{\mathrm{sd}}= & 2 \pi R_{\mathrm{M}}^{2} \rho\left(R_{\mathrm{M}}\right) c_{\mathrm{s}}^{3}\left(R_{\mathrm{M}}\right) \simeq \\
& 5.4 \times 10^{31} \dot{M}_{-6} a_{10 \mathrm{~d}}^{-2} v_{8}^{-1} R_{\mathrm{M} 10}^{1 / 2}\left(1+16 R_{\mathrm{a} 10} /\left(3 R_{\mathrm{M} 10}\right)\right)^{1 / 2} \mathrm{erg} \mathrm{s}^{-1} .
\end{aligned}
$$

In the above equation $R_{a 10}=10^{-10} R_{a}$ and $c_{s}\left(R_{M}\right)=v_{f f}\left(R_{M}\right)=\left(2 G_{N S} / R_{M}\right)^{1 / 2}$.

- The subsonic propeller regime: $\mathrm{R}_{\mathrm{M}}<\mathrm{R}_{\mathrm{a}}, \mathrm{R}_{\mathrm{co}}, \dot{M}_{\mathrm{w}}<\dot{M}_{\text {lim }}$. The break down of the supersonic propeller regime occurs when $\mathrm{R}_{\mathrm{M}}<\mathrm{R}_{\mathrm{co}}$, i.e., when the magnetosphere rotation is no longer supersonic with respect to the surrounding material. The structure of the atmosphere changes and the transition to the subsonic propeller regime takes place. Since the rotation of the magnetosphere is subsonic, the atmosphere is roughly adiabatic $(n=3 / 2)$, and the magnetospheric radius is approximated by (Davies \& Pringle, 1981):

$$
R_{\mathrm{M}} \simeq 2 \times 10^{10} a_{10 \mathrm{~d}}^{4 / 7} \dot{M}_{-6}^{-2 / 7} v_{8}^{8 / 7} \mu_{33}^{4 / 7} \mathrm{~cm} .
$$

In the subsonic propeller regime, the centrifugal barrier does not operate because $\mathrm{R}_{\mathrm{M}}<\mathrm{R}_{\mathrm{co}}$, but the energy input at the base of the atmosphere (due to NS rotational energy dissipation) is still too high for matter to penetrate the magnetosphere at a rate $\dot{M}_{\text {capt }}$ (Davies \& Pringle, 1981). Nevertheless a fraction of the matter inflow at $R_{a}$ is expected to accrete onto the NS, due to the KHI and Bohm diffusion ${ }^{4}$. We found that, in all the cases of interest to this paper, the KHI provides the largest contribution to the total luminosity in the subsonic propeller regime. By using similar arguments to those above, we estimated in this case

$$
L_{\mathrm{KH}}=1.8 \times 10^{35} \eta_{\mathrm{KH}} R_{\mathrm{M} 10}^{3} \dot{M}_{-6} \frac{\left(1+16 R_{\mathrm{a} 10} /\left(5 R_{\mathrm{M} 10}\right)\right)^{3 / 2}\left(\rho_{\mathrm{i}} / \rho_{\mathrm{e}}\right)^{1 / 2}}{P_{\mathrm{s} 3} a_{10 \mathrm{~d}}^{2} \nu_{8}\left(1+\rho_{\mathrm{i}} / \rho_{\mathrm{e}}\right)} \mathrm{erg} \mathrm{s}^{-1} .
$$

The subsonic propeller regime applies until the critical accretion rate

$$
\dot{M}_{\lim _{-6}}=2.8 \times 10^{2} P_{\mathrm{s} 3}^{-3} a_{10 \mathrm{~d}}^{2} v_{8} R_{\mathrm{M} 10}^{5 / 2}\left(1+16 R_{\mathrm{a} 10} /\left(5 R_{\mathrm{M} 10}\right)\right)^{-3 / 2}
$$

is reached, at which the gas radiative cooling (bremsstralhung) completely damps convective motions inside the atmosphere (Davies \& Pringle, 1981). If this cooling takes place, direct accretion at a rate $\dot{M}_{\text {capt }}$ onto the NS surface is permitted.

- The direct accretion regime: $\mathrm{R}_{\mathrm{M}}<\mathrm{R}_{\mathrm{a}}, \mathrm{R}_{\mathrm{co}}, \dot{M}_{\mathrm{w}}>\dot{M}_{\text {lim }}$. If $\mathrm{R}_{\mathrm{M}}<\mathrm{R}_{\text {co }}$ and matter outside the magnetosphere cools efficiently, accretion onto the NS takes place at the same rate $\dot{M}_{\text {capt }}$ (see Eq. 2.2) at which it flows towards the magnetosphere. The corresponding luminosity is

$$
L_{\mathrm{acc}}=G M_{\mathrm{NS}} \dot{M}_{\text {capt }} / R_{\mathrm{NS}}=2 \times 10^{35} \dot{M}_{-6} a_{10 \mathrm{~d}}^{-2} v_{8}^{-4} \mathrm{erg} \mathrm{s}^{-1} \simeq 2 \times 10^{35} \dot{M}_{15} \mathrm{erg} \mathrm{s}^{-1},
$$

where $\dot{M}_{15}=\dot{M}_{\text {capt }} / 10^{15} \mathrm{~g} \mathrm{~s}^{-1}$. This is the standard accretion regime; the system achieves the highest mass to luminosity conversion efficiency.

\footnotetext{
${ }^{4}$ To our knowledge this is the first application of the KHI to the subsonic propeller regime.
} 

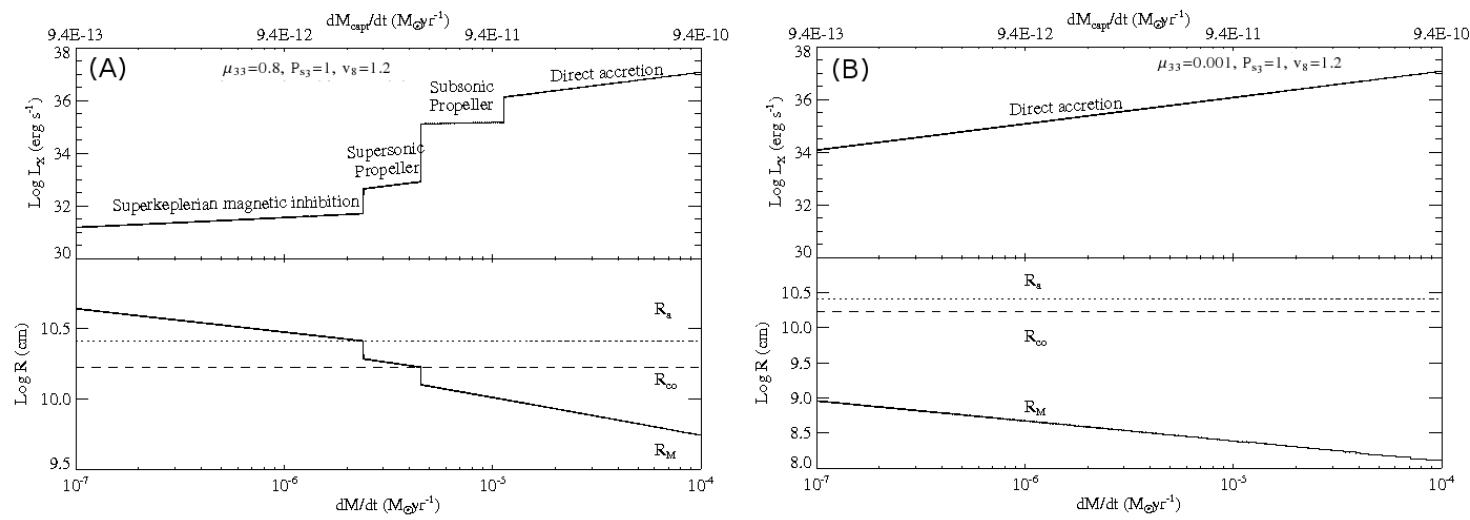

Figure 2: (A) Upper panel: Variation of the luminosity through different regimes, as a function of the mass loss rate from the companion star. In this case the parameters of the model are fixed at $\mu_{33}=0.8, \mathrm{P}_{\mathrm{s} 3}=1$, and $\mathrm{v}_{8}=1.2$. Lower panel: Relative position of the magnetospheric radius, $R_{M}$ (solid line), with respect to the accretion radius $R_{a}$ (dotted line), and the corotation radius $R_{c o}$ (dashed line), as a function of the mass loss rate from the companion star.

(B) Upper panel: Variation of the luminosity as a function of the mass loss rate from the companion star. In this case the parameters of the model are fixed at $\mu_{33}=0.001, \mathrm{P}_{\mathrm{s} 3}=1$, and $\mathrm{v}_{8}=1.2$. Lower panel: Relative position of the magnetospheric radius, $\mathrm{R}_{\mathrm{M}}$ (solid line), with respect to the accretion radius $\mathrm{R}_{\mathrm{a}}$ (dotted line), and corotation radius $\mathrm{R}_{\mathrm{co}}$ (dashed line), as a function of the mass loss rate from the companion star.

\section{Transitions and paths across different regimes}

We explore here the conditions under which transitions across different regimes take place. As emphasised in $\S 2$, these transitions occur when the relative positions of $R_{M}, R_{a}$, and $R_{c o}$ change in response to variations in the stellar wind parameters. In the following, since $\mathrm{R}_{\mathrm{M}}$ depends only weakly on the orbital period and the total mass of the system, we fix $\mathrm{a}_{10 \mathrm{~d}}=1$, and investigate variations in the other four parameters: $\mu_{33}, \mathrm{P}_{\mathrm{s} 3}, \mathrm{v}_{8}$, and $\dot{M}_{-6}$. The equations that define the conditions for transitions between different regimes are

$$
\begin{aligned}
& R_{\mathrm{M}}>R_{\mathrm{a}} \Rightarrow \dot{M}_{-6} \lesssim 0.45 \mu_{33}^{2} v_{8}^{11} a_{10 \mathrm{~d}}^{2} \text { magnetic barrier; } \\
& R_{\mathrm{M}}>R_{\mathrm{co}} \Rightarrow P_{\mathrm{s} 3} \lesssim 2.6 \dot{M}_{-6}^{-1 / 4} v_{8}^{-1 / 4} a_{10 \mathrm{~d}}^{1 / 2} \mu_{33}^{1 / 2} \text { centrifugal barrier with } R_{\mathrm{M}}>R_{\mathrm{a}} \text {; } \\
& R_{\mathrm{M}}>R_{\mathrm{co}} \Rightarrow P_{\mathrm{s} 3} \lesssim 1.8 a_{10 \mathrm{~d}}^{2 / 3} \dot{M}_{-6}^{-1 / 3} v_{8}^{2 / 3} \mu_{33}^{2 / 3} \text { centrifugal barrier with } R_{\mathrm{M}}<R_{\mathrm{a}} \text {; } \\
& P_{\mathrm{s} 3} \gtrsim 4.5 \dot{M}_{-6}^{-15 / 21} a_{10 \mathrm{~d}}^{30 / 21} v_{8}^{60 / 21} \mu_{33}^{16 / 21} \text { subsonic propeller } \rightarrow \text { direct accretion. }
\end{aligned}
$$

As an example, in Fig. 2A we use the above equations to compute the luminosity swings for a system with $\mu_{33}=0.8, \mathrm{P}_{\mathrm{s} 3}=1$, and $\mathrm{v}_{8}=1.2$. The lower panel of this figure shows that, for $0.1<\dot{M}_{-6}<100$, the magnetospheric radius crosses both the centrifugal $\left(\mathrm{R}_{\mathrm{co}}\right)$ and magnetic $\left(\mathrm{R}_{\mathrm{a}}\right)$ barriers. Correspondingly, the system moves from the superKeplerian magnetic inhibition regime, to the supersonic and subsonic propeller regime, and, finally, to the direct accretion regime, giving rise to a six-decade luminosity swing from $\sim 10^{31}$ to $\sim 10^{37} \mathrm{erg} \mathrm{s}^{-1}$. We note that a large part of this swing (about five decades) is attained across the transitions from the superKeplerian magnetic inhibition to the direct accretion regimes, which take a mere factor of $\sim 5$ variation of $\dot{M}_{\mathrm{w}}$.

Fig. 2B shows that, in the presence of a standard NS magnetic field $\left(10^{12} \mathrm{G}\right)$, such abrupt luminosity jumps are not expected for a very slowly rotating (1000 s) NS (the other system parameters 

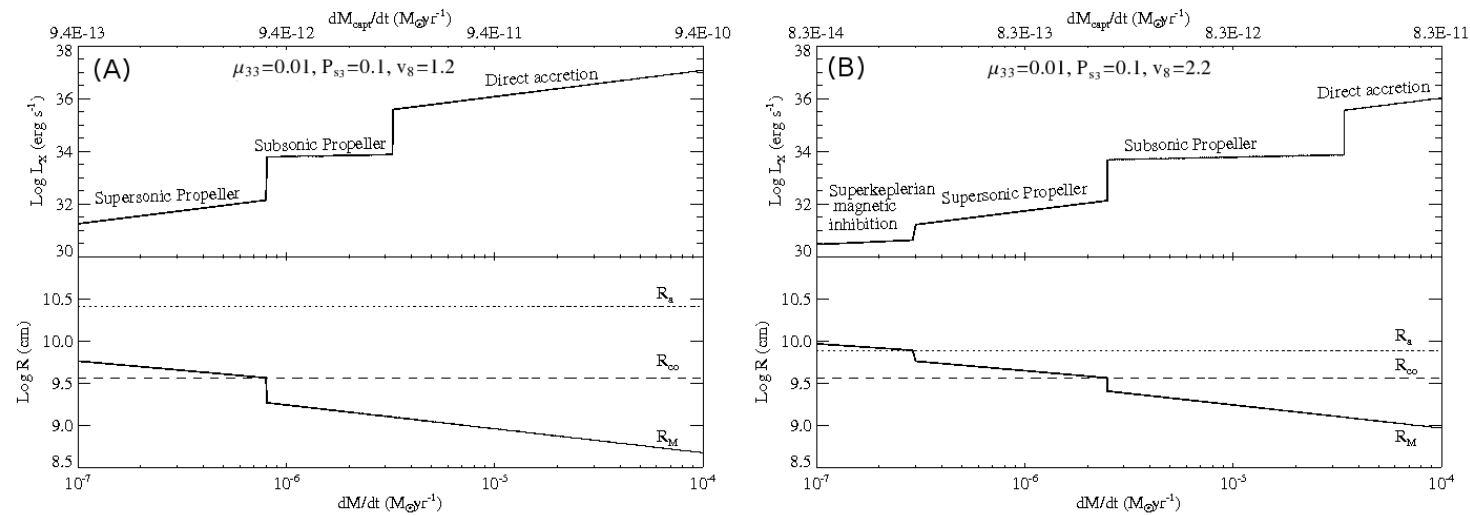

Figure 3: (A) Upper panel: Variation of the luminosity through different regimes, as a function of the mass loss rate. In this case the parameters of the model are fixed at $\mu_{33}=0.01, \mathrm{P}_{\mathrm{s} 3}=0.1$, and $\mathrm{v}_{8}=1.2$. Lower panel: Relative position of the magnetospheric radius, $R_{M}$ (solid line), with respect to the accretion radius $R_{a}$ (dotted line), and corotation radius $R_{c o}$ (dashed line), as a function of the mass loss rate from the companion star. (B) Same as (A) but for $\mu_{33}=0.01, \mathrm{P}_{\mathrm{s} 3}=0.1$, and $\mathrm{v}_{8}=2.2$.

are the same as those of Fig. $2 \mathrm{~A}$ ), since the magnetospheric radius is smaller than both $\mathrm{R}_{\mathrm{a}}$ and $\mathrm{R}_{\mathrm{co}}$, for any reasonable value of $\dot{M}_{\mathrm{w}}$. Therefore, the direct accretion regime applies, with the luminosity proportional to $\dot{M}_{\mathrm{w}}$.

In Fig. 3 we show the transitions for a system with $\mu_{33}=0.01$ and $\mathrm{P}_{\mathrm{s} 3}=0.1$. The wind velocity is $\mathrm{v}_{8}=1.2$ in Fig. $3 \mathrm{~A}$, and $\mathrm{v}_{8}=2.2$ in Fig. 3B. These two figures show that, for sub-magnetar fields, a 100 s spinning NS can undergo a transition across the magnetic barrier (besides the centrifugal barrier), for suitable parameters (a high wind velocity in the case at hand). Such transitions take place over a more extended interval of mass loss rates. For instance Fig. 3B shows that an increase by a factor $\sim 100$ in the mass loss rate is required, in this case, to achieve a factor $\sim 10^{5}$ luminosity swing; this is comparable with the magnetar case of Fig. 2A.

Taking into account of the examples discussed above, we conclude that:

- Long spin period systems $\left(P_{\mathrm{s} 3} \gtrsim 1\right)$ require magnetar-like B-fields $\left(\mu_{33} \geq 0.1\right)$ in order for a large luminosity swing $\left(\sim 10^{5}\right)$ to arise from modest variations in the wind parameters (e.g. a factor $\sim 5$ in $\dot{M}_{-6}$ ). These luminosity swings might result from transitions across different regimes through both the centrifugal and magnetic barriers.

- Shorter spin period systems $\left(P_{\mathrm{s} 3} \ll 1\right)$ must posses lower magnetic fields $\left(\mu_{33} \ll 0.1\right)$ for similar transitions to take place. Somewhat larger variations in the wind parameters are required in order to achieve similar luminosity swings to those of the long period case, and transitions between different regimes occur in most cases through the centrifugal barrier.

- Few or no transitions are expected for systems with either high magnetic fields and short spin periods, or systems with lower magnetic fields and long spin periods. In the first case the centrifugal barrier halts the inflowing matter at $R_{\mathrm{M}}$ and accretion does not take place; such systems might thus be observable only at very low (X-ray) luminosity levels $\left(\simeq 10^{32}-10^{33}\right.$ erg s$\left.^{-1}\right)$. In the second case $R_{\mathrm{M}}<R_{\mathrm{co}}$ for a wide range of wind parameters, accretion can take place, and a high persistent luminosity is released $\left(\simeq 10^{35}-10^{37}\right.$ erg s$\left.^{-1}\right)$. 


\section{Application to SFXT sources}

In this section we propose that transitions across different regimes caused by relatively mild variations of the wind parameters are responsible for the outbursts of SFXTs.

As a case study we consider IGR J17544-2619 (Sunyaev et al., 2003), a SFXT observed by Chandra during a complex transition to and from a $\sim 1$ hour-long outburst, yielding the first detailed characterization of a SFXT light curve over a wide range of luminosity. The spin period of IGR J17544-2619 is presently unknown. In't Zand (2005) showed that four different stages, with very different luminosity levels, could be singled out during the Chandra observation: (a) a quiescent state with $\mathrm{L}_{\mathrm{X}} \simeq 2 \times 10^{32} \mathrm{erg} \mathrm{s}^{-1}$, (b) a rise stage with $\mathrm{L}_{\mathrm{X}} \simeq 1.5 \times 10^{34} \mathrm{erg} \mathrm{s}^{-1}$, (c) the outburst peak with $\mathrm{L}_{X} \simeq 4 \times 10^{37} \mathrm{erg} \mathrm{s}^{-1}$, and (d) a post-outburst stage (or "tail") with $\mathrm{L}_{X} \simeq 2 \times 10^{36} \mathrm{erg} \mathrm{s}^{-1}$ (see panel (a) of Fig. 4; these luminosities are for a source distance of $\sim 3.6 \mathrm{kpc}$, Rahoui et al., 2008). The maximum luminosity swing observed across these stages was a factor of $\gtrsim 6.5 \times 10^{4}$.

Motivated by the evidence for $>1000$ s periodicities in XTE J1739-302 and IGR J16479-4514, we discuss first the possibility that IGR J17544-2619 contains a very slowly spinning NS. We use $\mu_{33}=1, \mathrm{P}_{\mathrm{s} 3}=1.3, \mathrm{v}_{8}=1.4$, and show in Fig. 4(b) the different regimes experienced by such a NS as a function of the mass loss rate. For $\dot{M}_{-6}<20$ the above values give $\mathrm{R}_{\mathrm{M}}>\mathrm{R}_{\mathrm{a}}$ and $\mathrm{R}_{\mathrm{M}}>\mathrm{R}_{\mathrm{co}}$, such that superKeplerian magnetic inhibition of accretion applies. The expected luminosity in this regime, $\sim 10^{31} \mathrm{erg} \mathrm{s}^{-1}$, is likely outshined by the $\mathrm{X}$-ray luminosity of the supergiant star (the companion star's luminosity is not shown in Fig. 4, but it is typically of order $\sim 10^{32} \mathrm{erg} \mathrm{s}^{-1}$, Cassinelli et al., 1981). We conclude that the lowest emission state (quiescence) of IGR J17544-2619 can be explained in this way, with the companion star dominating the high energy luminosity (In't Zand, 2005). The rise stage is in good agreement with the subKeplerian magnetic inhibition regime, where the luminosity $\left(\sim 10^{34} \mathrm{erg} \mathrm{s}^{-1}\right)$ is dominated by accretion of matter onto the NS due to the KHI. The uncertainty in the value of $h$ translates into an upper limit on the luminosity in this regime which is a factor of $\sim 10$ higher than that given above (Bozzo et al., 2008). During the outburst peak the direct accretion regime must apply at a mass loss rate of $\dot{M}_{-6}=500$. In this interpretation direct accretion must also be at work in the outburst tail at $\dot{M}_{-6} \sim 3$, where a slight decrease in $\dot{M}_{\mathrm{w}}$ would cause the magnetic barrier to close and the source to return to quiescence. According to this interpretation, if IGR J17544-2619 has a spin period of $>1000 \mathrm{~s}$, then it must host a magnetar.

Panel (c) of Fig. 4 shows an alternative interpretation of the IGR J17544-2619 light curve, where we fixed $\mu_{33}=0.08, \mathrm{P}_{\mathrm{s} 3}=0.4$, and $\mathrm{v}_{8}=1$. For this somewhat faster spin (and lower magnetic field), the luminosity variation is mainly driven by a transition across the centrifugal barrier (as opposed to the magnetic barrier). In this case, the quiescent state corresponds to the supersonic propeller regime $\left(\dot{M}_{-6}<0.6\right)$, the rise stage to the subsonic propeller $\left(0.6<\dot{M}_{-6}<2\right)$, while both the peak of the outburst and tail take place in the direct accretion regime at $\dot{M}_{-6}=200$ and $\dot{M}_{-6}=10$, respectively. Assuming an even faster NS spin period for IGR J17544-2619, a weaker magnetic field would be required. In panel (d) of Fig. 4, we show the results obtained by adopting $\mu_{33}=0.001$, $\mathrm{P}_{\mathrm{s} 3}=0.01$, and $\mathrm{v}_{8}=2$. The $\sim 10^{34} \mathrm{erg} \mathrm{s}^{-1}$ luminosity in the subsonic propeller regime compares well with the luminosity in the rise stage. However, the luminosity of the supersonic propeller regime is now significantly higher than the quiescence luminosity of $\sim 10^{32} \mathrm{erg} \mathrm{s}^{-1}$ (this is a consequence of the higher value of $\dot{M}_{\mathrm{w}}$ for which the supersonic propeller regime is attained in this interpretation). We note that, the whole luminosity swing takes place for a wider range of mass loss rates, and the 
outburst peak luminosity requires $\dot{M}_{-6} \simeq 3000$, an extremely high value even for an OB supergiant.

Interpreting the properties of IGR J17544-2619 in terms of a NS with a spin periods $\ll 100 \mathrm{~s}$ is more difficult. For instance, for the subsonic propeller regime to set in, the mass loss rate corresponding to the transition across $R_{M}=R_{c o}$ must be lower than the limit fixed by Eq. 2.14. If instead the transition takes place at higher mass loss rate, the system goes directly from the supersonic propeller to the direct accretion regime (or vice versa), bypassing the subsonic propeller: therefore, the rise stage would remain unexplained. Since fast rotating NSs require lower magnetic fields for direct accretion to take place while in outburst, Eq. 2.14 is satisfied only for very high wind velocities $\left(\mathrm{v}_{8}>2-3\right)$. On the other hand, an increase by a factor of $\sim 2$ in the wind velocity (with respect to the longer spin period solutions) would give a substantially lower $\dot{M}_{\text {capt }}$, such that the subsonic and the direct accretion regime luminosities fall shortwards of the observed values (unless unrealistically high mass loss rate are considered). Based on the above discussion, we conclude that IGR J17544-2619 likely hosts a slowly rotating NS, with spin period $>100$ s. Whether the magnetic barrier or the centrifugal barrier sets in, causing inhibition of accretion away from the outbursts, will depend on whether the spin period is longer or shorter then $\sim 1000 \mathrm{~s}$.

As another example we discuss the case of IGR J16465-4507, a SFXTs with a spin period of $228 \mathrm{~s}$. The luminosity behaviour of this source is still poorly known. An outburst at $5 \times 10^{36} \mathrm{erg} \mathrm{s}^{-1}$ was observed with INTEGRAL (assuming a distance of $12.5 \mathrm{kpc}$, Lutovinov et al., 2005; Smith, 2004), which did not detect the source before the outburst down to a level of $5 \times 10^{35} \mathrm{erg} \mathrm{s}^{-1}$. About a week later, XMM-Newton revealed the source at $5 \times 10^{34} \mathrm{erg} \mathrm{s}^{-1}$ and discovered the $228 \mathrm{~s}$ pulsations (Lutovinov et al., 2005). If the direct accretion regime applied all the way to the lowest luminosity level observed so far, then an upper limit of $\mu_{33} \simeq 0.004$ would be obtained by imposing that the NS did not enter the subsonic propeller regime. On the other hand, if the luminosity measured by XMM-Newton signalled that the source entered the subsonic propeller regime, while direct accretion occurred only during the outburst detected by INTEGRAL, then a considerably higher magnetic field of $\mu_{33} \simeq 0.07$ would be required.

The above discussion emphasizes the importance of determining, through extended high sensitivity observations, the luminosity at which transitions between different source states occur, in particular the lowest luminosity level for which direct accretion is still at work. In combination with the NS spin, this can be used to infer the NS magnetic field. Alternatively accretion might take place unimpeded at all luminosity levels of SFXTs, a possibility which requires a very clumpy wind as envisaged in other scenarios (Walter \& Zurita Heras, 2007). In this case the NS magnetic field can be considerably lower than discussed here.

\section{Conclusions}

In this paper we reviewed the theory of wind accretion in HMXBs hosting a magnetic NS with a supergiant companion, and considered in some detail the interaction processes between the inflowing plasma and the magnetosphere, that are expected to take place when direct accretion onto the NS surface is inhibited. We then applied this theory to SFXTs and showed that their large luminosity swings between quiescence and outburst (up to a factor of $\sim 10^{5}$ ) can be attained in response to relatively modest variations of the wind parameters, provided the system undergoes transitions across different regimes. We found that such transitions can be driven mainly by either: 

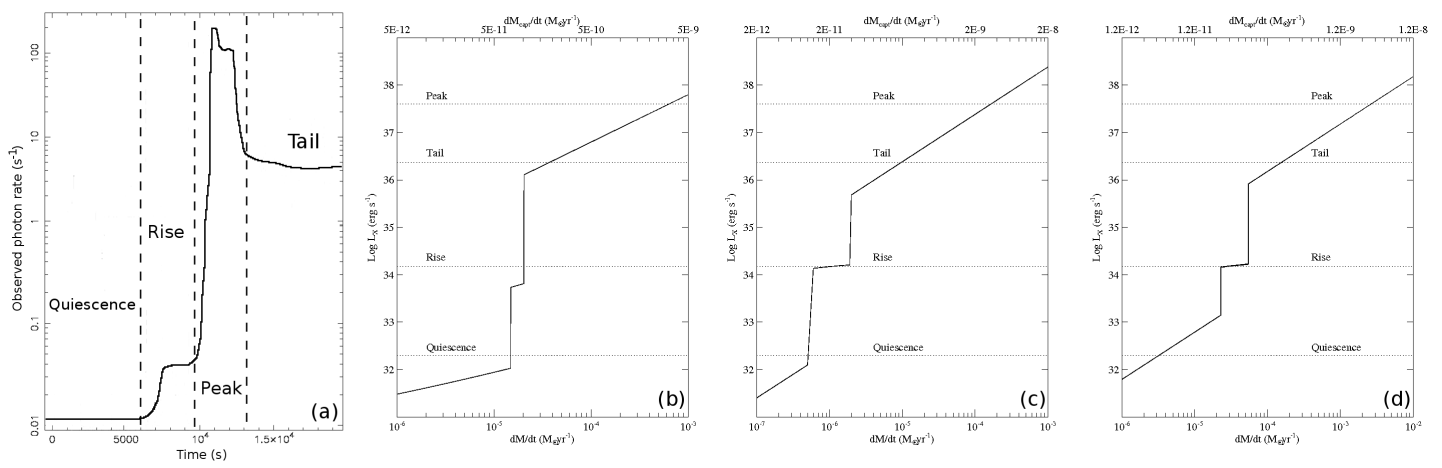

Figure 4: Application of the gated model to the IGR J17544-2619 transition from quiescence to outburst. Panel (a): A schematic representation of the Chandra light curve of IGR J17544-2619 obtained by using the segments that were not affected by pile-up (see both panels of figure 2 in In't Zand, 2005). The different luminosity stages are clearly visible. According to In't Zand (2005), the count rates on the y-axis correspond to $2 \times 10^{32} \mathrm{erg} \mathrm{s}^{-1}, 1.5 \times 10^{34} \mathrm{erg} \mathrm{s}^{-1}, 2 \times 10^{36} \mathrm{erg} \mathrm{s}^{-1}$, and $4 \times 10^{37} \mathrm{erg} \mathrm{s}^{-1}$, in the quiescence state, the rise state, the tail, and the peak of the outburst, respectively. Panel (b): Interpretation of the quiescence to outburst transition of IGR J17544-2619 in terms of the magnetic barrier model. The dotted horizontal lines mark the luminosity that divide the different regimes. The parameters of the model are fixed at $P_{\mathrm{s} 3}=1.3, v_{8}=1.4$, and $\mu_{33}=1$. Panel (c): Interpretation of the quiescence to outburst transition of IGR J17544-2619 based on the centrifugal barrier model. The parameters of the model are fixed at $\mu_{33}=0.08, P_{\mathrm{s} 3}=0.4$, and $v_{8}=1$. Panel (d): Same as panel (c) but here the parameters of the model are fixed at $\mu_{33}=0.001, P_{\mathrm{s} 3}=0.01$, and $v_{8}=2$.

(a) a centrifugal barrier mechanism, which halts direct accretion when the NS rotation becomes superKeplerian at the magnetospheric radius, or (b) a magnetic barrier mechanism, when the magnetosphere extends beyond the accretion radius. Which mechanism and wind interaction regime applies will depend sensitively on the NS spin period and magnetic field, besides the velocity and mass loss rate in the supergiant's wind. In particular, the magnetic barrier mechanism requires long spin periods ( $\gtrsim 1000 \mathrm{~s}$ ) coupled with magnetar-like fields ( $\left.\gtrsim 10^{14} \mathrm{G}\right)$. On the other hand, magnetarlike fields would also be required if the centrifugal barrier sets in at relatively high luminosities $\left(\gtrsim 10^{36} \mathrm{erg} \mathrm{s}^{-1}\right)$ in NSs with spin periods of hundreds seconds.

Evidence has been found that the spin periods of a few SFXTs might be as long as 1000-2000 s. Motivated by this, we presented an interpretation of the activity of IGR J17544-2619 (whose spin period is unknown) in terms of the magnetic barrier by a $1300 \mathrm{~s}$ spinning NS and showed that the luminosity stages singled out in a Chandra observation of this source are well matched by the different regimes of wind-magnetosphere interaction expected in this case. We discussed also an interpretation of this source based on the centrifugal barrier and a slightly shorter spin period (400 s), which can reproduce the luminosity stages comparably well. We emphasise that in both solutions the required magnetic field strength ( $\gtrsim 10^{15} \mathrm{G}$ and $\gtrsim 8 \times 10^{13} \mathrm{G}$, respectively) are in the magnetar range.

While the possibility that magnetars are hosted in binary system with supergiant companions has been investigated by several authors (e.g., Zhang et al., 2004; Liu \& Yan, 2006), clear observational evidence for such extremely high magnetic field NS in binary systems is still missing. According to the present study, long spin period SFXTs might provide a new prospective for detecting and studying magnetars in binary systems. 


\section{Acknowledgments}

EB thanks DTU Space, Technical University of Denmark and IASF-INAF for grant support for this conference. We thank an anonymous referee for his useful comments and suggestions. This work was supported through ASI and MIUR grant.

\section{References}

Bozzo, E., Falanga, M., Stella, L. 2008, ApJ, 683, 1031

Burnard, D. J., Arons, J., \& Lea, S. M. 1983, ApJ, 266, 175

Cassinelli, J. P., et al. 1981, ApJ, 250, 677

Davidson, K. \& Ostriker, J. P. 1979, ApJ, 179, 585

Davies, R. E., Fabian, A. C., \& Pringle, J. E. 1979, MNRAS, 186, 779

Davies, R. E. \& Pringle, J. E. 1981, MNRAS, 196, 209

Elsner, R. F. \& Lamb, F. K. 1977, ApJ, 215, 897

Frank J., et al. 2002, Accretion Power in Astrophysics (3th ed., Cambridge University Press)

Harding, A. K. \& Leventhal, M. 1992, Nature, 357, 388

Ikhsanov, N. R. \& Pustil'nik, L. A. 1996, A\&A, 312, 338

Illarionov, A. F. \& Sunyaev, R. A. 1975, A\&A, 39, 185

In 't Zand 2005, A\&A, 441, L1

Liu, Q. Z. \& Yan, J. Z. 2006, AdSpR, 38, 2906

Lutovinov, A., et al. 2005, A\&A, 444, 821

Negueruela, I., et al. 2008, in press [astro-ph/0801.3863]

Rahoui, F., Chaty, S., Lagage, P.-O., Pantin, E. 2008, A\&A, 484, 801

Sguera, V., et al. 2005, A\&A, 444, 221

Sguera, V., et al. 2006, ApJ, 646, 452

Sidoli, L., et al. 2007, A\&A, 476, 1307

Smith, D. M. 2004, Astr. Tel., 338

Stella, L., White, N. E., \& Rosner, R. 1986, ApJ, 308, 669

Sunyaev, R. A., et al. 2003, Astr. Tel., 190 
Toropina, O. D., Romanova, M. M., \& Lovelace, R. V. E. 2006, MNRAS, 371, 569

Walter, R., et al. 2006, A\&A, 453, 133

Walter, R. \& Zurita Heras, J. A. 2007, A\&A, 476, 335

Zhang, F., Li, X.-D. \& Wang, Z.-R. 2004, ChjAA, 4, 320 